\title{
ARITHMETIC OF POTTS MODEL HYPERSURFACES
}

\author{
MATILDE MARCOLLI AND JESSICA SU
}

\begin{abstract}
We consider Potts model hypersurfaces defined by the multivariate Tutte polynomial of graphs (Potts model partition function). We focus on the behavior of the number of points over finite fields for these hypersurfaces, in comparison with the graph hypersurfaces of perturbative quantum field theory defined by the Kirchhoff graph polynomial. We give a very simple example of the failure of the "fibration condition" in the dependence of the Grothendieck class on the number of spin states and of the polynomial countability condition for these Potts model hypersurfaces. We then show that a period computation, formally similar to the parametric Feynman integrals of quantum field theory, arises by considering certain thermodynamic averages. One can show that these evaluate to combinations of multiple zeta values for Potts models on polygon polymer chains, while silicate tetrahedral chains provide a candidate for a possible occurrence of non-mixed Tate periods.
\end{abstract}

\section{INTRODUCTION}

A lot of attention was devoted in recent years to the intriguing occurrence of periods of algebraic varieties and motives in the context of perturbative quantum field theory and residues of Feynman integrals. The question initially arose from numerical observations of Broadhurst and Kreimer [12] on the occurrence of multiple zeta values in the computation of Feynman diagrams of massless scalar field theories. Multiple zeta values were conjectured to be the periods of mixed Tate motives, a result recently proved by Francis Brown [13. Moreover, the parametric formulation of Feynman integral (see [9, 24]) exhibits the residues of Feynman diagrams explicitly as periods of an algebraic variety (the graph hypersurface complement), at least modulo divergences and renormalization. It was then natural to expect that the occurrence of multiple zeta values would be an indication of the fact that the graph hypersurfaces are mixed Tate motives. This was conjectured by Kontsevich, in a substantially equivalent statement predicting that these hypersurfaces would be polynomially countable. This means that their counting of points over finite fields would depend polynomially on the order of the field. At first this conjecture was confirmed (see [35, [36]) for all graphs with up to twelve edges, but it was later disproved by a very elegant and general result of Belkale and Brosnan [7, which shows that, contrary to expectations, the classes of the graph hypersurfaces span a suitable localization of the Grothendieck ring of varieties, hence they can be arbitrarily far from the mixed Tate case as motives. For a discussion of the difference between the behavior of the classes in the localized or in the ordinary Grothendieck ring see also 2]. The original cases observed numerically in [12] were later confirmed to be periods of mixed Tate motives in [14, [15, [33] and other cases in which the parametric Feynman integral turns out to be a period of a mixed Tate motive were analyzed in [3], 10, [1], 18, 32, 33, and others. 
While the result of Belkale and Brosnan [7 that disproved the Kontsevich conjecture is very general and elegant, it is not immediately constructive, in the sense that it does not exhibit an explicit graph that fails to satisfy the polynomial countability property. The first such explicit example was identified by Doryn in [17, and other examples were then constructed in [16. There are interesting conceptual differences between these examples, which point to two different forms in which the polynomial countability property can fail. One, as in the case of [17, where the dependence in the order of the field fails to satisfy the same polynomial function at all primes, and another more drastic one, where an actual non-polynomial function appears.

In the recent paper [1], it was shown that some of the techniques employed in the study of the classes of the graph hypersurfaces in the Grothendieck ring of varieties can be extended to another type of physical model, this time not in quantum field theory but in statistical mechanics. In fact, the partition function for the Potts model for a spin system on a graph with $q$ possible states is given by a combinatorial polynomial associated to the graph, the multivariate Tutte polynomial, see 34. This is closely related to the Kirchhoff polynomial that defines the graph hypersurfaces in quantum field theory. In the context of Potts models, one is less directly interested in the periods of the hypersurface complement, and more in describing how the hypersurface behaves when over families of graphs that approximate some infinite graph. In fact, zeros of the partition function correspond to physical phase transitions, if they happen for non-negative real values of the parameters, while they can be thought of as some kind of non-physical "virtual" phase transitions otherwise. While (at least in the ferromagnetic case) the Potts model on a finite graph does not have phase transitions, which can only appear in the limit of an infinite graph, in the antiferromagnetic case there are possible phase transitions also over a finite graph 34. The sets of real zeros of the multivariate Tutte polynomials have been extensively studied in the Potts model literature, most notably by Jackson and Sokal 25. In 1 it is shown that one can estimate how the complexity of this set of zeros grows in certain families of graphs that approach infinite graphs, in terms of classes in the Grothendieck ring of the varieties defined by the multivariate Tutte polynomials, the Potts model hypersurfaces. These are analyzed using a deletion-contraction type formula similar to the one obtained in [4] for the graph hypersurfaces of quantum field theory.

In the present paper, continuing to draw on the analogy between the graph hypersurfaces and the Potts model hypersurfaces, $\mathcal{Z}_{G, q}$, for a graph $G$ and for a number $q$ of spin states, we compare the behavior of the counting of points over finite fields for these varieties. We first consider the "fibration condition" discussed in [1, that is, the question of whether the Grothendieck class of $\mathcal{Z}_{G, q}$ depends on $q$ outside of the special values $q=0$ and $q=1$. This question is motivated by an observation made in 1, where it was shown that, for certain classes of graphs (such as polymer chains), the classes of the hypersurfaces $\mathcal{Z}_{G, q}$ behave as if the hypersurfaces were a locally trivial fibration away from those two special values. We show here that the fibration condition already fails for the tetrahedron graph $K 4$, and we argue that one can use a Monte Carlo method, similar to the one developed in 27, [23], to test the failure of the fibration condition for Potts model hypersurfaces of more complicated graphs. We then look at the polynomial countability question for the Potts model hypersurfaces. Naturally, one expects that failures of polynomial 
countability will appear for much smaller and combinatorially simpler graphs than in the cases of the graph hypersurfaces of [16] and [17]. Indeed, this is the case. A trivial kind of failure of polynomial countability comes from the primes that divide the number of spin states $q$, but even after reformulating the question by taking into account this trivial failure, we see that, again, the tetrahedron graph $K 4$ already provides an example where a more interesting failure of polynomial countability is possible.

We discuss the role of periods in this statistical mechanical setting, where we interpret them in terms of certain thermodynamic averages. Unlike the more difficult case of quantum field theories, where infrared divergences arise from the intersection between the locus of integration in the parameteric Feynman integral and the graph hypersurfaces, in the (ferromagnetic) Potts model case the hypersurface does not intersect the simplex over which the integration is performed, hence we can directly interpret the resulting integral as a period, without the serious additional complication of blowups performing regularization and renormalization, as one finds ([10], [11]) in the quantum field theory case.

The main purpose of this paper is pedagogical. It is aimed at presenting this approach to Potts models through the algebro-geometric and motivic properties of the hypersurfaces defined by the multivariate Tutte polynomials, in terms of very concrete and simple examples, which, we hope, can serve as an illustration of the general methodology.

\section{Graph hypersurfaces and Potts model hypersurfaces}

To fix notation, in the following we denote by $G=G(V, E)$ a finite graph, with vertex set $V$ and edge set $E$. We also write $V=V(G)$ and $E=E(G)$.

2.1. Quantum field theory and graph hypersurfaces. We first recall briefly the definition of the graph hypersurfaces of perturbative quantum field theory.

Definition 2.1. Let $G=G(V, E)$ be a graph with $n=\# V$. Assign an edge weight (a variable) $t_{e}$ to each edge $e \in E$. The Kirchhoff graph polynomial of $G$ is of the form

$$
\Psi_{G}\left(t_{1}, \ldots, t_{n}\right)=\sum_{T \subset G} \prod_{e \notin E(T)} t_{e}
$$

where the sum is over all the spanning trees $T$ (or maximal spanning forests in the multi-connected case). The graph hypersurface $X_{G}$ is the hypersurface in $\mathbb{A}^{n}$ defined by the vanishing of the graph polynomial

$$
\mathcal{X}_{G}=\left\{t=\left(t_{1}, \ldots, t_{n}\right) \in \mathbb{A}^{n} \mid \Psi_{G}(t)=0\right\} .
$$

Since the graph polynomial is homogeneous of degree $b_{1}(G)$, the affine graph hypersurface is the affine cone over a projective graph hypersurface in $\mathbb{P}^{n-1}$.

The role of the graph hypersurfaces in perturbative quantum field theory is explained by the following result (see [9], 24], 29]). The Feynman integral associated to the graph $G$ is given by

$$
U(G)=\int_{\sigma_{n}} \frac{P_{G}(p, t)^{-n+D(n-1) / 2}}{\Psi_{G}(t)^{n(-1+D / 2)}} \omega_{n},
$$


where $D$ is the spacetime dimension, $P_{G}$ is the second graph polynomial (whose explicit form we do not recall here), with $p$ the external momenta, $\sigma_{n}$ the unit simplex, and $\omega_{n}$ the volume form. Here the Feynman integral refers to the residue (after removing a divergent Gamma factor), written in momentum space and in the Feynman parametric form, for the case of a massless scalar field theory (see the references listed above for more details). Modulo important issues of divergence and renormalization, the integral $(2.3)$ is then a period of the graph hypersurface complement.

2.2. Statistical mechanics and Potts model hypersurfaces. The analogous object we will be discussing in the statistical mechanics setting is the Potts model partition function.

To avoid notational ambiguity, we will write $\mathfrak{p}$ for an integer prime and $\mathfrak{q}=\mathfrak{p}^{n}$ for a prime power, so that we will be using the notation $\mathbb{F}_{\mathfrak{q}}$ is the finite field with $\mathfrak{q}$ elements. We reserve the notation $q$ for the variable in the Potts model partition function that denotes the number of spin states.

Definition 2.2. Let $G=G(V, E)$ be a graph with $n=\# V$, and with edge weights $t=\left(t_{e}\right)_{e \in V}$. The multivariate Tutte polynomial of $G$ is

$$
T_{G}(q, t)=\sum_{A \subseteq E} q^{k(A)} \prod_{e \in A} t_{e}
$$

where the sum is over the subgraphs of $G$ that span all vertices (that is, subgraphs $G^{\prime}$ with $V\left(G^{\prime}\right)=V(G)$ and $E\left(G^{\prime}\right)=A \subset E$ ), and where $k(A)$ is the number of connected components in each subgraph, and $q$ is an indeterminate.

To see the relation to Potts models in statistical mechanics, we consider a set $\mathfrak{A}$ of cardinality $q$ (here assumed to be a non-negative integer) of possible spin states, and a statistical system of spins assigned to the vertices of a graph $G$. A state of the spin system if an assignment of a spin state $\sigma_{v}$ to each vertex. The energy of a state is the sum over all edges of a quantity

$$
H(e)=\left\{\begin{array}{cc}
0 & \sigma_{v} \neq \sigma_{w} \\
-J_{e} & \sigma_{v}=\sigma_{w},
\end{array}\right.
$$

where $\partial(e)=\{v, w\}$, and where $J_{e}$ is a fixed value, with $J_{e} \geq 0$ in the ferromagnetic case and $-\infty \leq J_{e} \leq 0$ in the antiferromagnetic case. The weight edges are then related to the energies $J_{e}$ by the relation

$$
t_{e}=e^{\beta J_{e}}-1,
$$

where $\beta$ is (up to the Boltzmann constant) an inverse temperature. The partition function of the Potts model statistical system on $G=G(V, E)$ is then given by the expression

$$
Z_{G}(q, t)=\sum_{\sigma: V \rightarrow \mathfrak{A}} \prod_{e \in E}\left(1+t_{e} \delta_{\sigma_{v}, \sigma_{w}}\right),
$$

where the sum is over all states (that is, all maps of vertices to spin states), $\delta$ is the Kronecker delta, and $\partial(e)=\{v, w\}$. The relation of the multivariate Tutte polynomial (2.4) to statistical mechanics comes from a famous result of FortuinKasteleyn [20], which shows that the partition function of the Potts model is a 
restriction of the multivariate Tutte polynomial

$$
Z_{G}(q, t)=\left.T_{G}(q, t)\right|_{q \in \mathbb{N}, t=e^{\beta J}-1 \in \mathbb{R}} .
$$

The Potts model hypersurface is the locus of zeros defined by the multivariate Tutte polynomial

$$
\mathcal{Z}_{G}=\left\{(q, t) \in \mathbb{A}^{n+1} \mid Z_{G}(q, t)=0\right\} .
$$

Notice that, unlike the Kirchhoff graph polynomial, the multivariate Tutte polynomial is not homogeneous, so this is an affine hypersurface in $\mathbb{A}^{n+1}$, where $n=$ $\# E(G)$, but not a projective hypersurface. One also considers the Potts model hypersurface for fixed $q$,

$$
\mathcal{Z}_{G, q}=\left\{t \in \mathbb{A}^{n} \mid Z_{G}(q, t)=0\right\} .
$$

The relation between the Potts model hypersurfaces and the graph hypersurfaces of quantum field theory lies in the fact (see [1, 34]) that the Kirchhoff graph polynomial $\Psi_{G}$, or rather its equivalent form

$$
\Phi_{G}(t)=\sum_{T \subset G} \prod_{e \in E(T)} t_{e},
$$

which is related to $\Psi_{G}$ by dividing by $\prod_{e \in E} t_{e}$ and applying the Cremona transformation $t_{e} \mapsto 1 / t_{e}$ to all the edge variables, is obtained by normalizing the $\tilde{T}_{G}(q, t)=q^{-k(G)} T_{G}(q, t)$, evaluating at $q=0$ and taking the homogeneous piece of lowest degree. This relation has a more geometric interpretation in terms of tangent cones, as in Lemma 2.7 of 11. This relation is the motivation for extending techniques developed in the quantum field theory context for studying the geometry of the graph hypersurfaces to the setting of Potts models.

2.3. Counting points and classes in the Grothendieck ring. Let $\mathcal{V}_{\mathbb{K}}$ denote the category of (quasi-projective) algebraic varieties over a field $\mathbb{K}$. The Grothendieck ring of varieties $K_{0}\left(\mathcal{V}_{\mathbb{K}}\right)$ is the free abelian group generated by the isomorphic classes $[X]$ of varieties, modulo the relations

$$
[X]=[Y]+[X \backslash Y]
$$

for closed embeddings $Y \subset X$ of subvarieties. The product operation that gives the ring structure is given by $[X] \cdot[Y]=[X \times Y]$.

For an affine hypersurface $X \subset \mathbb{A}^{n}$, we will use in the following the notation $\{X\}$ for the class in $K_{0}\left(\mathcal{V}_{\mathbb{K}}\right)$ of the hypersurface complement

$$
\{X\}:=\left[\mathbb{A}^{n} \backslash X\right]=\left[\mathbb{A}^{n}\right]-[X]=\mathbb{L}^{n}-[X],
$$

where $\mathbb{L}=\left[\mathbb{A}^{1}\right]$ is the Lefschetz motive, the class of the affine line.

Similarly, one can define a Grothendieck ring $K_{0}\left(\mathcal{V}_{\mathbb{Z}}\right)$ for arithmetic varieties defined over $\mathbb{Z}$. While the relations are formally the same, the classes $[X]$ in this case denote isomorphism classes as varieties over $\mathbb{Z}$. We will sometime write only $K_{0}(\mathcal{V})$, without specifying the field or ring of definition, when it should be clear from the context.

An additive invariant is a map $\chi: \mathcal{V}_{\mathbb{K}} \rightarrow R$, with values in a commutative ring $R$, that satisfies $\chi(X)=\chi(Y)$ if $X$ and $Y$ are isomorphic, and $\chi(X)=\chi(Y)+\chi(X \backslash Y)$, for closed embeddings $Y \subset X$. Moreover, one requires that $\chi(X \times Y)=\chi(X) \chi(Y)$. An additive invariant determines and is determined by a ring homomorphism $\chi$ : 
$K_{0}\left(\mathcal{V}_{\mathbb{K}}\right) \rightarrow R$. For varieties in $\mathcal{V}_{\mathbb{C}}$, the topological Euler characteristic is an additive invariant, and the properties of being additive under decompositions $X=Y \cup(X \backslash$ $Y)$ and multiplicative under products are its defining properties, hence one can consider the class $[X]$ in the Grothendieck ring as a universal Euler characteristic of the variety $X$, see 8 .

Recall that for every prime number $\mathfrak{p}$ and every $n \geq 1$ there is a unique field extension $\mathbb{F}_{\mathfrak{q}}$ of degree $n$ of $\mathbb{F}_{\mathfrak{p}}$, with $\mathfrak{q}=\mathfrak{p}^{n}$. In the case of varieties $X$ defined over a finite field $\mathbb{F}_{\mathfrak{p}}$. One denotes then by $X\left(\mathbb{F}_{\mathfrak{p}^{m}}\right)$ the set of $\mathbb{F}_{\mathfrak{p}^{m}}$-points of $X$, for $m \geq 1$. One then sets

$$
N_{\mathfrak{p}^{m}}(X)=\# X\left(\mathbb{F}_{\mathfrak{p}^{m}}\right),
$$

the number of $\mathbb{F}_{\mathfrak{p}^{m}}$-points of $X$. Then it is not hard to see that $N_{\mathfrak{p}^{m}}(X)$ is an additive invariant of $X$, hence it factors through $K_{0}\left(\mathcal{V}_{\mathbb{F}_{\mathfrak{p}}}\right)$, so that we can write $N_{\mathfrak{p}^{m}}([X])$ as a function of the class $[X]$ in the Grothendieck ring.

In the following, we will be considering varieties $X$ defined over $\mathbb{Z}$. In this case, we can either regard them as complex varieties by embedding $\mathbb{Q} \subset \mathbb{C}$, or reduce modulo $\mathfrak{p}$, at the various primes, and obtain varieties $X_{\mathfrak{p}}$ defined over $\mathbb{F}_{\mathfrak{p}}$, for which one can consider the counting of points $N_{\mathfrak{p}^{m}}(X)$.

We are interested in the behavior of the number of points $N_{\mathfrak{q}}(X)$ as a function of $\mathfrak{q}$. We recall the following terminology (see [7, 35, 36]).

Definition 2.3. Let $X$ be a variety over $\mathbb{Z}$ with reduction $X_{\mathfrak{q}}$ over $\mathbb{F}_{\mathfrak{q}}$, for $\mathfrak{q}=\mathfrak{p}^{m}$. Then $X$ is polynomially countable if $N_{\mathfrak{q}}\left(X_{\mathfrak{q}}\right)$ is a polynomial function of $\mathfrak{q}$.

Because the counting of points over finite fields is an additive invariant, which factors through the Grothendieck ring of varieties, one can use polynomial countability as a test for the motivic nature of the hypersurfaces, as follows.

If $\mathbb{L}=\left[\mathbb{A}^{1}\right]$ is the Lefschetz motive in $K_{0}(\mathcal{V})$, then $\mathbb{Z}[\mathbb{L}] \subset K_{0}(\mathcal{V})$ is the subring of the Tate motives. More precisely, one should view the Tate motives as $\mathbb{Z}\left[\mathbb{L}, \mathbb{L}^{-1}\right] \subset$ $K_{0}(\mathcal{M})$, for $\mathcal{M}$ the category of pure Chow motives, but for our purposes it suffices to work with $K_{0}(\mathcal{V})$. There is, anyway, a homomorphism $\chi_{m o t}: K_{0}(\mathcal{V}) \rightarrow K_{0}(\mathcal{M})$ defined by the additive invariant of [22. For varieties that are not smooth and projective, one leaves the category of pure motives and one needs to consider objects in a more complicated (triangulated) category of mixed motives, which contains a (triangulated) subcategory of mixed Tate motives. However, even varieties that are singular still define classes in the Grothendieck ring, and the $\mathbb{Z}[\mathbb{L}]$ part of the Grothendieck ring contains the classes of the varieties whose motive is a mixed Tate motive.

Then, if a variety $X$ defined over $\mathbb{Z}$ is a mixed Tate motive, it has class $[X] \in \mathbb{Z}[\mathbb{L}]$ in $K_{0}\left(\mathcal{V}_{\mathbb{Z}}\right.$ ). Away from up to finitely many primes $\mathfrak{p}$ (where some bad reduction phenomenon can occur), it then follows that $N_{\mathfrak{p}^{m}}\left(X_{\mathfrak{p}}\right)$ is a polynomial in $\mathfrak{p}^{m}$, by the fact that the counting $N_{\mathfrak{p}^{m}}\left(X_{\mathfrak{p}}\right)$ factors through the Grothendieck ring $K_{0}\left(\mathcal{V}_{\mathbb{F}_{\mathfrak{p}}}\right)$ and that $N_{\mathfrak{p}^{m}}\left(\mathbb{A}^{1}\right)=\mathfrak{p}^{m}$. Thus, if a variety $X$ does not have the property of being polynomially countable at all but finitely many primes, it follows that it is not a mixed Tate motive.

In fact, polynomial countability at all but finitely many primes is conjecturally equivalent to the motive of the variety being mixed Tate: the Tate conjecture predicts that determining $N_{\mathfrak{p}}\left(X_{\mathfrak{p}}\right)$ for almost all primes $\mathfrak{p}$ would determine the motive of $X$, see $[6$. 


\section{THE FIBRATION CONDITION}

In [1] a specific condition for the classes in the Grothendieck ring of the Potts model hypersurfaces is identified, called the "fibration condition", according to which the the Grothendieck class $\left\{\mathcal{Z}_{G}\right\}$ of the complement of the hypersurface $\mathcal{Z}_{G}$ behaves as one would expect in the case of a fibration on the locus $q \neq 0,1$. This means that the class $\left\{\mathcal{Z}_{G, q}\right\}$ is independent of $q$, for all $q \neq 0,1$, and that

$$
\left\{\mathcal{Z}_{G}\right\}=(\mathbb{T}-1) \cdot\left\{\mathcal{Z}_{G, q \neq 0,1}\right\}+\mathbb{T}^{\# E(G)}
$$

This condition is satisfied by the families of graphs considered in [1, but the general question of whether it holds for more general graphs was not addressed in that paper, nor was the question of whether, when the condition on the Grothendieck classes is satisfied, the variety $\mathcal{Z}_{G}$ is really a locally trivial fibration over the locus $q \neq 0,1$.

Here we show that, in fact, very simple examples of graphs already do not satisfy the fibration condition and the number of points over $\mathbb{F}_{\mathfrak{p}}$ of $\mathcal{Z}_{G, q}$ has a nontrivial dependence on $q \neq 0,1$.

Proposition 3.1. The graph $K 4$ does not satisfy the fibration condition.

Proof. One can see this by a direct computation of the number of points over $\mathbb{F}_{\mathfrak{p}}$ for different values of $q$. The following table illustrates the failure of the fibration condition.

\begin{tabular}{|c|c||c|}
\hline $\mathfrak{p}$ & $q$ & $\# \mathcal{Z}_{G, q}\left(\mathbb{F}_{\mathfrak{p}}\right)$ \\
\hline \hline 11 & 0 & 1771561 \\
\hline 11 & 1 & 771561 \\
\hline 11 & 2 & 173799 \\
\hline 11 & 3 & 173183 \\
\hline 11 & 4 & 173821 \\
\hline 11 & 5 & 173513 \\
\hline 11 & 6 & 174151 \\
\hline 11 & 7 & 173227 \\
\hline 11 & 8 & 173447 \\
\hline 11 & 9 & 173579 \\
\hline 11 & 10 & 173799 \\
\hline
\end{tabular}

By comparison, polygons are the simplest example of graphs that do satisfy the fibration condition of [1]. In fact, by Proposition 5.2 of [1] we know that for a polygon $C_{m+1}$ with $m+1$ sides, one has

$$
\left\{\mathcal{Z}_{C_{m+1}, q \neq 0,1}\right\}=\mathbb{T}^{m+1}+\mathbb{T}\left(\mathbb{T}^{m}-(\mathbb{T}-1)^{m}\right)+\frac{(\mathbb{T}-1)^{m}-(-1)^{m}}{\mathbb{T}},
$$

with $\mathbb{T}=\mathbb{L}-1$, and it is therefore independent of $q \neq 0,1$. Comparing this expression with Proposition 5.1 of [1, one sees that indeed (3.1) holds for the polygons. 


\section{Monte Carlo method For COUnting points}

Monte Carlo methods for counting points of varieties over finite fields were introduced in [23] and [27]. We employ here the algorithm described in [23] and we show how it compares with the deterministic counting of points, on a sufficiently simple example of a graph where both computations can be performed. We use again the example of the tetrahedron graph $K_{4}$, which we already discussed in the previous section.

The following tables provides an explicit comparison between the deterministic counting and the Monte Carlo method (after 10000, 40000, and 100000 trials, respectively) for $K_{4}$, in the case $q=2$.

\begin{tabular}{|c||c|c|c|}
\hline $\mathfrak{p}$ & Monte Carlo & \% error & \% error bound \\
\hline \hline 3 & 413.9262 & 0.002242615 & 0.03363242 \\
\hline 5 & 4507.8125 & 0.013219263 & 0.047440384 \\
\hline 7 & 20670.9293 & -0.011007641 & 0.060059259 \\
\hline 11 & 179459.1293 & -0.004845872 & 0.079343222 \\
\hline 13 & 396763.6998 & -0.015535761 & 0.087605918 \\
\hline 17 & 1469977.952 & -0.015716002 & 0.101770275 \\
\hline 19 & 2399339.931 & -0.070218248 & 0.108087334 \\
\hline 23 & 6928079.605 & 0.045288885 & 0.119636754 \\
\hline \hline 3 & 412.1037 & -0.002170218 & 0.01681621 \\
\hline 5 & 4453.125 & 0.000927175 & 0.023720192 \\
\hline 7 & 21141.5253 & 0.011507837 & 0.03002963 \\
\hline 11 & 182825.0952 & 0.013819407 & 0.039671611 \\
\hline 13 & 404848.6049 & 0.004524793 & 0.043802959 \\
\hline 17 & 1503167.109 & 0.006507159 & 0.050885138 \\
\hline 19 & 2560472.073 & -0.007777023 & 0.054043667 \\
\hline 23 & 6661615.005 & 0.005085466 & 0.059818377 \\
\hline \hline 3 & 413.74395 & 0.001801332 & 0.010635505 \\
\hline 5 & 4477.5 & 0.006405934 & 0.015001967 \\
\hline 7 & 20710.92996 & -0.009093825 & 0.018992405 \\
\hline 11 & 183480.5728 & 0.017454225 & 0.02509053 \\
\hline 13 & 402024.9216 & -0.00248143 & 0.027703424 \\
\hline 17 & 1499908.538 & 0.004325248 & 0.032182587 \\
\hline 19 & 2532009.315 & -0.018806787 & 0.034180216 \\
\hline 23 & 6580195.266 & -0.007198912 & 0.037832464 \\
\hline & & & \\
\hline
\end{tabular}

The values should be compared with the deterministic values reported in the table in Proposition 5.8. The expected error bound is computed as in [23], with an error margin $\epsilon$ of $\sqrt{4 b(\log (2 / \delta)) / N}$, where $b$ is the fraction of points that are roots, $N$ is the number of trials, and $1-\delta$ is the probability of being within $\epsilon$ of the correct fraction.

The Monte Carlo method is useful to test properties such as the fibration condition, when direct deterministic computations become intractable. In fact, when using the Monte Carlo method in cases with non-constant $q$-dependence, the deviance from a constant value is typically greater than what allowed by the error estimate. 


\section{FAilures of POLYNOMial COUNTABILITy}

We now analyze failures of polynomial countability for the Potts model hypersurfaces, which confirms the fact that these hypersurfaces are much less likely to be mixed Tate motives than the graph hypersurfaces of quantum field theory, although families of mixed Tate cases can still be constructed, using the deletion-contraction relation derived in 1, in cases where a good recursive procedure is possible, such as the polymer chains analyzed in 1 .

We use a variant of the algorithm used by Stembridge [36] in the case of the graph hypersurfaces. This can be adapted to our setting, because some general results proved in 36 apply both to the graph polynomials and the multivariate Tutte polynomials, in particular those discussed in Lemma 5.6 below.

5.1. The special case $q=1$. There is a very special case, which can be treated separately by direct geometric reasoning, which is the case with $q=1$. Notice that this is not a physically relevant case, since $q$ is the number of spin states.

When $q=1$, the multivariate Tutte polynomial assumes the very simple form

$$
\left.Z_{G}(q, t)\right|_{q=1}=\sum_{A \subset E} \prod_{e \in A} t_{e}=\prod_{e \in E}\left(1+t_{e}\right) .
$$

In fact, multiplying this out gives all possible combinations of the $t_{e}$ 's, which correspond to all possible subsets $A$ of $E$.

Lemma 5.1. In the case $q=1$, the counting of points over $\mathbb{F}_{\mathfrak{p}}$ is given by

$$
N_{\mathfrak{p}}\left(\mathcal{Z}_{G, q=1}\right)=\mathfrak{p}^{\# E(G)}-(\mathfrak{p}-1)^{\# E(G)} .
$$

Proof. The edge weight tuple satisfies the Tutte polynomial iff at least one of the edge weights $t_{e}$ is -1 . Therefore, we can get the number of roots by taking the total number of tuples and subtracting the number of tuples where none of the edge weights is -1 . If there are $\mathfrak{p}$ elements in the field and $m$ edges, the number of roots is $\mathfrak{p}^{m}-(\mathfrak{p}-1)^{m}$. Therefore, the number of roots always depends polynomially on $\mathfrak{p}$ when $q=1$.

One can also see this directly from the class $\left[\mathcal{Z}_{G, q=1}\right]$ in the Grothendieck ring, as observed in [1, the locus of zeros of the polynomial $\prod_{e \in E}\left(1+t_{e}\right)$ is isomorphic to the union of coordinate hyperplanes, whose complement in $\mathbb{A} \# E(G)$ is a torus, whose class is $\mathbb{T}^{\# E(G)}$. Thus, the class of the complement is $\left\{\mathcal{Z}_{G, q=1}\right\}=\mathbb{T}^{\# E(G)}$ and $\left[\mathcal{Z}_{G, q=1}\right]=\mathbb{L}^{\# E(G)}-\mathbb{T}^{\# E(G)}$, which immediately gives back the formula of Lemma 5.1. since $N_{\mathfrak{p}}(\mathbb{L})=\mathfrak{p}$ and $N_{\mathfrak{p}}(\mathbb{T})=\mathfrak{p}-1$.

5.2. A trivial failure for $q \neq 1$. We now consider the case where $q \neq 1$ (we also assume $q \neq 0$ ). In this case, we show that there is always a "trivial" failure of polynomial countability, which has to do with the primes that divide $q$. In fact, in the way it is originally written, the multivariate Tutte polynomial has an overall multiplicative factor of $q^{k(G)}$, with $k(G)$ the number of connected components of $G$. Thus, whenever $\mathfrak{p}$ is a prime that divides $q$, the polynomial becomes identically zero in the reduction mod $\mathfrak{p}$.

We start with the following simple observation. 
Lemma 5.2. If a set of points satisfies a single variable polynomial at infinitely many values but does not satisfy that polynomial at certain other values, the entire set of points cannot satisfy a single variable polynomial (even if it is a different polynomial).

Proof. Suppose given an infinite set $\mathcal{S}$ with an infinite subset $\mathcal{T} \subset \mathcal{S}$, such that the points of $\mathcal{T}$ satisfy a polynomial $Q(x)$ of degree $m$ and the points of $\mathcal{S}$ satisfy a polynomial $P$ of degree $n$, then these two polynomials must be the same. In fact, both polynomials have to agree on the infinite set $\mathcal{T}$, hence their difference $R(x)=P(x)-Q(x)$ is a polynomial with infinitely many zeros, that is, the trivial polynomial $R \equiv 0$.

We then have the following general behavior.

Corollary 5.3. For a fixed value $q \neq 0,1$ of the number of spin states, let $G$ be a graph for which the class $\left[\mathcal{Z}_{G, q}\right]$ in $K_{0}\left(\mathcal{V}_{\mathbb{Z}}\right)$ is in $\mathbb{Z}[\mathbb{L}]$. Then the counting function $N_{\mathfrak{p}}\left(\mathcal{Z}_{G, q}\right)$ is given by

$$
N_{\mathfrak{p}}\left(\mathcal{Z}_{G, q}\right)= \begin{cases}\mathfrak{p}^{\# E(G)} & \mathfrak{p} \mid q \\ P_{q}(\mathfrak{p}) & \mathfrak{p} \backslash q\end{cases}
$$

where $\left[\mathcal{Z}_{G, q}\right]=P_{q}(\mathbb{L})=a_{0}+a_{1} \mathbb{L}+\cdots+a_{N} \mathbb{L}^{N}$ in $\mathbb{Z}[\mathbb{L}]$. Thus, $N_{\mathfrak{p}}\left(\mathcal{Z}_{G, q}\right)$ is not a polynomial function.

Proof. If $q=0$ in the field (that is, if $\mathfrak{p}$ is a divisor of $q$ ), everything is a root, so there are $\mathfrak{p}^{m}$ roots where $m$ is the number of edges. If $q \neq 0$ ( $\mathfrak{p}$ does not divide $q$ ), by the form $\left[\mathcal{Z}_{G, q}\right]=P_{q}(\mathbb{L})=a_{0}+a_{1} \mathbb{L}+\cdots+a_{N} \mathbb{L}^{N}$ of the class in the Grothendieck ring we obtain that $N_{\mathfrak{p}}\left(\mathcal{Z}_{G, q}\right)=P_{q}(\mathfrak{p})$. The last observation then follows from Lemma 5.2

Notice that this type of failure of the polynomial countability, due only to the primes that divide the number $q$ of spin states, should be regarded as an "accidental" and not a "serious" failure of polynomial countability, in the sense that, for example, it does not really affect the nature of the classes in the Grothendieck ring.

We can see this explicitly in some simple examples. Consider first the case where $G=T$ is a tree. Then the multivariate Tutte polynomial is of the form

$$
Z_{T}(q, t)=q \prod_{e \in E(T)}\left(q+t_{e}\right) .
$$

Example 5.4. The counting function $N_{\mathfrak{p}}\left(\mathcal{Z}_{T_{m}, q}\right)$ for a tree $T_{m}$ with $m$ edges is given by

$$
N_{\mathfrak{p}}\left(\mathcal{Z}_{T_{m}, q}\right)= \begin{cases}\mathfrak{p}^{m} & \mathfrak{p} \mid q \\ \mathfrak{p}^{m}-(\mathfrak{p}-1)^{m} & \mathfrak{p} \backslash q\end{cases}
$$

Another very simple explicit example is that of polygons, for which the expression (3.2) for the class in the Grothendieck ring gives the following.

Example 5.5. The counting function $N_{\mathfrak{p}}\left(\mathcal{Z}_{C_{m+1}, q}\right)$ for a polygon $C_{m+1}$ is given by $\mathfrak{p}^{m+1}$ when $\mathfrak{p} \mid q$, while when $\mathfrak{p} \backslash$, it is given by

$$
N_{\mathfrak{p}}\left(\mathcal{Z}_{C_{m+1}, q}\right)=\mathfrak{p}^{m+1}-\left((\mathfrak{p}-1)^{m+1}+(\mathfrak{p}-1)\left((\mathfrak{p}-1)^{m}-(\mathfrak{p}-2)^{m}\right)+\frac{(\mathfrak{p}-2)^{m}-(-1)^{m}}{(\mathfrak{p}-1)}\right) .
$$


Another way to see that $N_{\mathfrak{p}}\left(\mathcal{Z}_{C_{m+1}, q}\right)$ is a polynomial in $\mathfrak{p}$, for all $\mathfrak{p}$ that do not divide $q$, which does not use directly the expression 3.2 for the class in the Grothendieck ring, is to show, as in Stembridge 36 that a partial deletioncontraction relation holds at the level of the counting functions. This reflects the partial deletion-contraction relation at the level of classes in the Grothendieck ring proved in [1].

5.3. Probabilistic counting functions. We recall the relevant notation from [36. Given a set of polynomials $\left\{f_{1}, \ldots, f_{k}\right\}$ in $\mathbb{Z}\left[x_{1}, \ldots, x_{m}\right]$, one writes $\mathfrak{Z}\left[f_{1}, \ldots, f_{k}\right]$ for the probability that all the $f_{i}$ vanish at a uniformly randomly chosen $\left(x_{1}, \ldots, x_{m}\right) \in$ $\mathbb{F}_{\mathfrak{q}}$. Then, as a function of the field cardinality $\mathfrak{q}=\mathfrak{p}^{r}$, one has

$$
\mathfrak{Z}\left[f_{1}, \ldots, f_{k}\right](\mathfrak{q})=\mathfrak{q}^{-m} N_{\mathfrak{q}}\left(X_{\mathfrak{p}}\right),
$$

where $X_{\mathfrak{p}}$ is the reduction $\bmod \mathfrak{p}$ of the variety defined over $\mathbb{Z}$ by the polynomials $\left\{f_{1}, \ldots, f_{k}\right\}$ and $N_{\mathfrak{q}}$ is the number of its $\mathbb{F}_{\mathfrak{q}}$-points, for $\mathfrak{q}$ a power of $\mathfrak{p}$. Then by Proposition 2.1 of [36], the variety $X$ is polynomially countable if and only if $\mathfrak{Z}\left[f_{1}, \ldots, f_{k}\right](\mathfrak{q})$ is a polynomial in $\mathfrak{q}^{-1}$. We will refer to $\mathfrak{Z}\left[f_{1}, \ldots, f_{k}\right]$ as the "probabilistic counting function".

Then we have the following analog of Lemma 3.2 of [36].

Lemma 5.6. Let $Z_{G, q}=Z_{G}(q, t)$ denote the multivariate Tutte polynomial, for fixed $q \neq 0,1$, as a function of the edge weights $t=\left(t_{e}\right)$, and let $\mathfrak{Z}\left[Z_{G, q}\right]$ be the corresponding probabilistic counting function. Then this satisfies the relation

$$
\mathfrak{Z}\left[Z_{G, q}\right](\mathfrak{p})=\mathfrak{p}^{-1}-\mathfrak{p}^{-1} \mathfrak{Z}\left[Z_{G / e, q}\right]+\mathfrak{Z}\left[Z_{G / e, q}, Z_{G \backslash e, q}\right]
$$

Proof. The multivariate Tutte polynomials satisfy a deletion-contraction relation

$$
Z_{G}(q, t)=Z_{G \backslash e}\left(q, \hat{t}^{(e)}\right)+t_{e} Z_{G / e}\left(q, \hat{t}^{(e)}\right)
$$

for any edge $e$ (regardless of whether it is a regular edge, a bridge, or a looping edge), with $\hat{t}^{(e)}$ denoting the set of edge weights with $t_{e}$ removed, and where $G / e$ is the graph obtained by contracting $e$ in $G$ and $G \backslash e$ the graph obtained by deleting $e$ in $G$.

Then the argument of Proposition 2.3 of [36] and the deletion-contraction relation (5.7) show that one obtains the relation $(5.6)$.

A more refined deletion-contraction relation of a similar nature to (5.6) was proved by algebro-geometric methods in [1], for classes in the Grothendieck ring. Namely, for the classes of the hypersurface complements one has

$$
\left\{\mathcal{Z}_{G, q}\right\}=\mathbb{L} \cdot\left\{\mathcal{Z}_{G / e, q} \cap \mathcal{Z}_{G \backslash e, q}\right\}-\left\{\mathcal{Z}_{G / e, q}\right\} .
$$

One sees then that one can also recover 5.6 directly from (5.8). Indeed, we have from (5.8), that (away from primes that divide $q$ ) the counting function satisfies

$$
\begin{aligned}
\left.N_{\mathfrak{p}}\left(\mathbb{A}^{\# E(G)} \backslash \mathcal{Z}_{G, q}\right)\right) & \left.=\mathfrak{p} N_{\mathfrak{p}}\left(\mathbb{A}^{\# E(G)-1} \backslash\left(\mathcal{Z}_{G / e, q} \cap \mathcal{Z}_{G \backslash e, q}\right)\right)\right) \\
& \left.-N_{\mathfrak{p}}\left(\mathbb{A}^{\# E(G)-1} \backslash \mathcal{Z}_{G / e, q}\right)\right)
\end{aligned}
$$

which gives

$$
\left.N_{\mathfrak{p}}\left(\mathcal{Z}_{G, q}\right)\right)=\mathfrak{p} N_{\mathfrak{p}}\left(\mathcal{Z}_{G / e, q} \cap \mathcal{Z}_{G \backslash e, q}\right)+\mathfrak{p}^{\# E(G)-1}-N_{\mathfrak{p}}\left(\mathcal{Z}_{G / e, q}\right)
$$

Thus, one obtains, as expected,

$$
\mathfrak{Z}\left[Z_{G, q}\right](\mathfrak{p})=\mathfrak{p}^{-\# E(G)} N_{\mathfrak{p}}\left(\mathcal{Z}_{G, q}\right)=\mathfrak{p}^{-1}+\mathfrak{Z}\left[\mathcal{Z}_{G / e, q} \cap \mathcal{Z}_{G \backslash e, q}\right]-\mathfrak{p}^{-1} \mathfrak{Z}\left[\mathcal{Z}_{G / e, q}\right]
$$


Similarly, one can define another probabilistic counting function, which we denote here by $\mathfrak{Z}^{\vee}\left[f_{1}, \ldots, f_{k}\right]=1-\mathfrak{Z}\left[f_{1}, \ldots, f_{k}\right]$. This is the probability of not having a common zero of the $f_{i}$. Just as $\mathfrak{Z}\left[f_{1}, \ldots, f_{k}\right](\mathfrak{q})$ is related to the number of points $N_{\mathfrak{q}}\left(X_{\mathfrak{p}}\right)$ by $\mathfrak{Z}\left[f_{1}, \ldots, f_{k}\right](\mathfrak{q})=\mathfrak{q}^{-m} N_{\mathfrak{q}}\left(X_{\mathfrak{p}}\right)$, the probabilistic counting function $\mathfrak{Z}^{\vee}\left[f_{1}, \ldots, f_{k}\right]$ satisfies

$$
\mathfrak{Z}^{\vee}\left[f_{1}, \ldots, f_{k}\right](\mathfrak{q})=\mathfrak{q}^{-m}\left(\mathfrak{q}^{m}-N_{\mathfrak{q}}\left(X_{\mathfrak{p}}\right)\right)=\mathfrak{q}^{-m} N_{\mathfrak{q}}\left(\mathbb{A}^{m} \backslash X_{\mathfrak{p}}\right) .
$$

In other words, $\mathfrak{Z}^{\vee}\left[f_{1}, \ldots, f_{k}\right]$ is the probabilistic counting function of the variety's complement. Thus, from (5.8) we have

$$
\mathfrak{Z}^{\vee}\left[Z_{G, q}\right]=\mathfrak{Z}^{\vee}\left[Z_{G \backslash, q}, Z_{G / e, q}\right]-\mathfrak{q}^{-1} \mathfrak{Z}^{\vee}\left[Z_{G / e, q}\right] .
$$

5.4. The case of the graph $K 4$. We now focus, in particular, on the polynomial countability question for the case of the tetrahedron graph $K 4$, and we show that it exhibits a more serious failure of polynomial countability, which is not only due to the primes that divide $q$.

Lemma 5.7. $\mathfrak{Z}\left[x^{2}+2 x+2\right]$ is not a polynomial.

Proof. First observe that there are infinitely many primes of the form $\mathfrak{p}=4 k+$ 3. This follows from Dirichlet's theorem, showing more generally that there are infinitely many primes in any arithmetic progression. (For this particular case there is also a direct elementary proof, which we do not report here.) Then notice that, for all primes $\mathfrak{p}$ of the form $\mathfrak{p}=4 k+3$, the polynomial $x^{2}+2 x+2$ has no solutions over $\mathbb{F}_{\mathfrak{p}}$. In fact, by the quadratic formula, $x^{2}+2 x+2$ has a solution if and only if $x=\frac{-2 \pm \sqrt{4-8}}{2}=-1 \pm \sqrt{-1}$. This has a solution iff $\sqrt{-1} \in \mathbb{F}_{\mathfrak{p}}$. Assume this is true, and that $\mathfrak{p}=4 k+3$. Let $a=\sqrt{-1}$, then we have $a^{\mathfrak{p}-1}=a^{4 k+2}=\left(a^{2}\right)^{2 k+1}=$ $-1^{2 k+1}=-1$, but by Fermat's Little Theorem, $a^{\mathfrak{p}-1} \equiv 1$. Therefore, $x^{2}+2 x+2$ has no solution for all primes of the form $\mathfrak{p}=4 k+3$. Now, if $\mathfrak{Z}\left[x^{2}+2 x+2\right]$ were a polynomial, then it would have infinitely many zeros, since there are infinitely many primes $\mathfrak{p}$ (those of the form $\mathfrak{p}=4 k+3$ ), where $x^{2}+2 x+2$ has no solution, but $\mathfrak{Z}\left[x^{2}+2 x+2\right]$ is not identically zero: for instance, when $\mathfrak{p}=5$, there are two roots, $x=1$ and $x=2$. Thus, $\mathfrak{Z}\left[x^{2}+2 x+2\right]$ is not polynomial.

Consider then the graph $K 4$ and in the case with $q=2$ (Ising model). A direct calculation based on a version of the algorithm of Stembridge gives

$$
\begin{aligned}
N_{\mathfrak{p}}\left(\mathcal{Z}_{K 4, q=2}\right)= & \mathfrak{p}^{5} \mathfrak{Z}[P(x)]+2 \mathfrak{p}^{3} \mathfrak{Z}\left[2+2 x_{4}+x_{4}^{2}\right] \\
& +2 \mathfrak{p}^{3} \mathfrak{Z}\left[2+2 x_{5}+x_{5}^{2}\right]+\mathfrak{p}^{5}-\mathfrak{p}^{4}-3 \mathfrak{p}^{3}+13 \mathfrak{p}^{2}-\mathfrak{p}-1 \\
& -\mathfrak{p}^{5} \mathfrak{Z}[2, P(x)]-2 \mathfrak{p}^{3} \mathfrak{Z}\left[2,2+2 x_{4}+x_{4}^{2}\right]-2 \mathfrak{p}^{3} \mathfrak{Z}\left[2,2+2 x_{5}+x_{5}^{2}\right] \\
& +\mathfrak{Z}[2]\left(\mathfrak{p}^{6}-\mathfrak{p}^{5}+\mathfrak{p}^{4}+3 \mathfrak{p}^{3}-13 \mathfrak{p}^{2}+\mathfrak{p}+1\right),
\end{aligned}
$$

where the polynomial $P(x)$ is of the form

$$
\begin{aligned}
P(x) & =4 x_{2} x_{4} x_{5}+8 x_{2} x_{3} x_{4} x_{5}+4 x_{2} x_{3} x_{4}+4 x_{2} x_{3} x_{5}+4 x_{3} x_{4} x_{5} \\
& +4 x_{3} x_{4}+4 x_{2} x_{5}+2 x_{3} x_{4}^{2}+2 x_{3}^{2} x_{4}+x_{3}^{2} x_{4}^{2}+2 x_{2} x_{3}^{2} x_{4} \\
& +x_{2} x_{3}^{2} x_{4}^{2}+2 x_{2} x_{3} x_{4}^{2}+2 x_{3} x_{4}^{2} x_{5}+2 x_{3}^{2} x_{4} x_{5}+x_{3}^{2} x_{4}^{2} x_{5} \\
& +2 x_{2}^{2} x_{4} x_{5} x_{3}+2 x_{2} x_{4}^{2} x_{5} x_{3}+x_{3}^{2} x_{4}^{2} x_{5} x_{2}+2 x_{3}^{2} x_{4} x_{5} x_{2} \\
+ & 2 x_{2}^{2} x_{5}+2 x_{2}^{2} x_{4} x_{5}+2 x_{2}^{2} x_{5} x_{3}+2 x_{2} x_{5}^{2}+x_{2}^{2} x_{5}^{2}+x_{2}^{2} x_{4} x_{5}^{2}
\end{aligned}
$$




$$
+2 x_{2} x_{4} x_{5}^{2}+x_{2}^{2} x_{3} x_{5}^{2}+2 x_{2} x_{3} x_{5}^{2}+x_{2}^{2} x_{4} x_{5}^{2} x_{3}+2 x_{2} x_{4} x_{5}^{2} x_{3} .
$$

The $\mathfrak{Z}[2]$ terms are only 1 in the case where $2=0$, which we can ignore as we are looking for non-trivial failures of polynomial countability. Among the remaining terms, we have seen in Lemma 5.7 that the $\mathfrak{Z}\left[2+2 x+x^{2}\right]$ contribute non-polynomial expressions. This strongly suggests that $\mathfrak{Z}\left[Z_{K 4, q}\right]$ itself may be non-polynomial. However, one needs to make sure that there are no cancellations (however unlikely) coming from the term $\mathfrak{p}^{5} \mathfrak{Z}[P(x)]$.

This is confirmed also by looking directly at the number of points for sufficiently many primes, as follows.

Proposition 5.8. For $q=2$ (Ising model case), the function $N_{\mathfrak{p}}\left(\mathcal{Z}_{K 4, q=2}\right)$ is nonpolynomial.

Proof. The values for the first few primes $\mathfrak{p} \neq 2$ give the following table.

\begin{tabular}{|c||c|c|c|c|c|c|c|c|}
\hline $\mathfrak{p}$ & 3 & 5 & 7 & 11 & 13 & 17 & 19 & 23 \\
\hline$N_{\mathfrak{p}}$ & 413 & 4449 & 20901 & 180333 & 403025 & 1493449 & 2580541 & 6627909 \\
\hline
\end{tabular}

Proposition 2.2 of [36] shows that, since the graph $K_{4}$ has six edges, if $N_{\mathfrak{p}}\left(\mathcal{Z}_{K 4, q=2}\right)$ is given by a polynomial, then this polynomial would have degree at most five. Thus, one obtains from the table above a system of linear equations for the coefficients of this polynomial,

$$
a_{0}+\mathfrak{p} a_{1}+\mathfrak{p}^{2} a_{2}+\mathfrak{p}^{3} a_{3}+\mathfrak{p}^{4} a_{4}+\mathfrak{p}^{5} a_{5}=N_{\mathfrak{p}},
$$

for $\mathfrak{p}$ and $N_{\mathfrak{p}}$ in the table above. Already when solving the first five equations in this system, one finds only a solution with rational, non-integer values of the $a_{i}$. By Proposition 6.1 of [31], one knows that if $N_{\mathfrak{p}}\left(\mathcal{Z}_{K 4, q=2}\right)$ is polynomial with rational coefficients then the coefficients must in fact be integers. Indeed, if one further considers the remaining three equations from the table of values above, one finds that there are no solutions. Thus, $N_{\mathfrak{p}}\left(\mathcal{Z}_{K 4, q=2}\right)$ is non-polynomial.

While the result of Proposition 5.8 alone suffices to show that the Potts model hypersurface of the graph $K 4$ is not polynomially countable, we have included the previous discussion to illustrate a possible explicitly source of non-polynomial terms.

5.5. Normalized Tutte polynomial. We make a small additional remark on the trivial failures of polynomial countability. In order to avoid the presence of an overall factor $q^{k(G)}$ in the multivariate Tutte polynomial, one sometimes considers, instead of the polynomial 2.4 , the normalized version

$$
\tilde{Z}_{G}(q, t)=q^{-k(G)} Z_{G}(q, t) .
$$

The effect of this change on the counting function is only to alter it at the primes $\mathfrak{p}$ that divide the number of states $q$. For example, in the two simple examples of $C_{4}$ and $K_{4}$, one finds the following.

Example 5.9. For the normalized polynomial (5.13), the counting function for $C_{4}$ with $q=2$ is given by

$$
p^{3}-3 p^{2} \mathfrak{Z}[2]+5 p^{2}+5 p \mathfrak{Z}[2]-7 p-1,
$$

which differs from the one for the Tutte polynomial (2.4) by

$$
\mathfrak{Z}[2]\left(-p^{4}+p^{3}+2 p^{2}-2 p-1\right) .
$$


For the graph $K_{4}$ with $q=2$ the counting function for the normalized case gives

$$
\begin{gathered}
p^{5} \mathfrak{Z}[P(x)]+2 p^{3} \mathfrak{Z}\left[2+2 x_{4}+x_{4}^{2}\right]+2 p^{3} \mathfrak{Z}\left[x_{5}^{2}+2 x_{5}+2\right] \\
+p^{5}-p^{4}-3 p^{3}+13 p^{2}-p-1+\mathfrak{Z}[2]\left(2 p^{3}-15 p^{2}+2 p\right),
\end{gathered}
$$

which again differs from the case of the original Tutte polynomial only at $\mathfrak{p}=2$, with the difference given by

$$
\begin{gathered}
\mathfrak{Z}[2]\left(-p^{6}+p^{5}-p^{4}-p^{3}-2 p^{2}+p-1\right)+p^{5} \mathfrak{Z}[2, P(x)] \\
+2 p^{3} \mathfrak{Z}\left[2, x_{4}^{2}+2 x_{4}+2\right]+2 p^{3} \mathfrak{Z}\left[2, x_{5}^{2}+2 x_{5}+2\right] .
\end{gathered}
$$

\section{THERMODYNAMIC AVERAGES AND PERIODS}

Coming back to the physical interpretation of the multivariate Tutte polynomial $Z_{G}(q, t)$ as the partition function of the Potts model with $q$ spin states on the graph $G$, the edge weights are of the form $t_{e}=\exp \left(\beta J_{e}\right)-1$, where $J_{e} \geq 0$ is the energy (in the ferromagnetic case) and $\beta$ is the inverse temperature. One knows that, for a finite graph and in the ferromagnetic case, there are no phase transitions, that is, no zeros of $Z_{G}(q, t)$ that fall in the range $t_{e} \geq 0$ and the phase transitions only appear in the limit $n \rightarrow \infty$ of a family of finite graphs $G_{n}$ approximating an infinite graph (see [34]).

Given a function $f(J)=F(t)$ of the energies $J=\left(J_{e}\right)$, or of the edge weights $t=\left(t_{e}\right)$, one can compute the thermodynamic average

$$
\langle F\rangle=\frac{\sum_{A \subseteq E} q^{k(A)} F\left(t_{A}\right) \prod_{e \in A} t_{e}}{\sum_{A \subseteq E} q^{k(A)} \prod_{e \in A} t_{e}}=\frac{1}{Z_{G}(q, t)} \sum_{A \subseteq E} q^{k(A)} F\left(t_{A}\right) \prod_{e \in A} t_{e},
$$

where we write $F\left(t_{A}\right)=\left.F(t)\right|_{t_{e}=0, \forall e \notin A}$. We are especially interested here in the case where $F(t)$ is a polynomial function of the edge variables, with rational coefficients.

Moreover, we can further average over a range of energies (at a fixed temperature), for example, by letting the edge variables range over the simplex $\Delta=\{t=$ $\left.\left(t_{e}\right) \mid t_{e} \geq 0, \sum_{e} t_{e}=1\right\}$. One then finds an expression that is formally very similar to the parametric Feynman integral in perturbative quantum field theory, namely

$$
\frac{1}{\operatorname{Vol}(\Delta)} \int_{\Delta}\langle F\rangle d v=\frac{1}{\operatorname{Vol}(\Delta)} \int_{\Delta} \frac{P_{G, F}(q, t)}{Z_{G}(q, t)} d v(t),
$$

where we write $P_{G, F}(q, t)$ for the polynomial

$$
P_{G, F}(q, t)=\sum_{A \subseteq E} q^{k(A)} F\left(t_{A}\right) \prod_{e \in A} t_{e},
$$

under the assumption that $F$ is itself a polynomial.

The normalization factor in (6.1) can be computed easily: the volume of a regular $n$-dimensional simplex with side length $a$ is given by the well known expression

$$
\operatorname{Vol}\left(\Delta_{n}(a)\right)=\frac{a^{n}}{n !} \sqrt{\frac{n+1}{2^{n}}} .
$$

Thus, the interesting number, whose nature one wants to investigate, is the remaining expression

$$
\int_{\Delta} \frac{P_{G, F}(q, t)}{Z_{G}(q, t)} d v(t)
$$




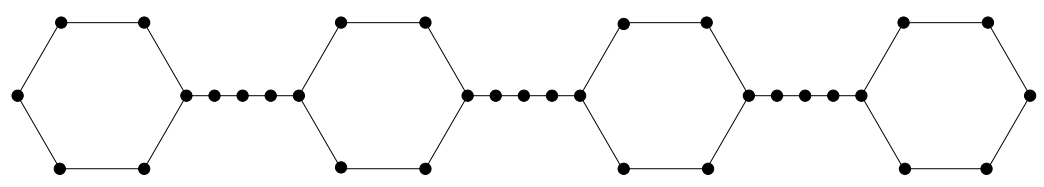

Figure 1. A polygon polymer chain (from [1]).

We see that the expression 6.3 that we obtain in this way is formally similar to the parametric Feynman integral (although with a very different physical interpretations), with $Z_{G}(q, t)$ the multivariate Tutte polynomial instead of the graph polynomial, and with $P_{G, F}(q, t)$ instead of the second Symanzik polynomial.

An interesting difference with respect to the quantum field theory case, which makes the Potts model case nicer, is that (as recalled above) the partition function $Z_{G}(q, t)$, for a finite graph $G$ does not have any zeros in the domain of integration. Thus, these integrals are genuine periods and are not plagued by the infrared divergences problem that occurs in their quantum field theoretic analogs, where the graph hypersurface intersects the simplex $\Delta$.

By analogy with the context of quantum field theory, one can then ask the question of what kind of numbers one obtains by evaluating the integrals (6.3), for arbitrary polynomials $F(t)$ with rational coefficients.

The integral $(6.3)$ is a period of the variety $\mathbb{A}^{n} \backslash \mathcal{Z}_{G, q}$, where $n=\# E(G)$ : the integral of an algebraic differential form over a domain of integration given by a (semi)algebraic set defined by algebraic equations. Periods are a very interesting class of numbers, see the detailed account given in 28 .

In particular, what kind of numbers can arise as periods of a given variety depends on the nature of the motive of the variety. For example, it was long conjectured and recently proved in 13 that the periods of mixed Tate motives over $\mathbb{Z}$ are $\mathbb{Q}\left[\frac{1}{2 \pi i}\right]$-linear combinations of multiple zeta values. The latter are expressions of the form

$$
\zeta\left(n_{1}, \ldots, n_{r}\right)=\sum_{0<k_{1}<\ldots<k_{r}} \frac{1}{k_{1}^{n_{1}} \cdots k_{r}^{n_{r}}},
$$

with integers $n_{i} \geq 1$ and $n_{r} \geq 2$.

Thus, for example, in the cases of polygon polymer chains considered in [1, where it is shown by the explicit formula (3.2) that the motives involved are mixed Tate, one can conclude that the periods (6.3) must evaluate to combinations of multiple zeta values, which is not obvious from the definition of the integral.

We assume here some standard conjectures about motives, in particular the Tate conjecture which implies that determining $N_{\mathfrak{p}}(X)$ for almost all primes $\mathfrak{p}$ determines the motive of $X$ (see [6], §1.1).

Proposition 6.1. Let ${ }^{(m, k)} G_{N}$ be a chain of linked polygons, obtained by joining $N$ polygons, each with $m+1$ sides and connected by a chain of $k$ egdes, as illustrated in Figure 1 . Then the thermodynamic averages $(6.3)$ are $\mathbb{Q}\left[\frac{1}{2 \pi i}\right]$-linear combinations of multiple zeta values. 
Proof. The explicit formula given in Proposition 5.5 of [1] for the class in the Grothendieck ring gives

$$
\left\{\mathcal{Z}_{(m, k)} G_{N}, q\right\}=\mathbb{T}^{k(N-1)} \cdot\left\{\mathcal{Z}_{C_{m+1}, q}\right\},
$$

where $\left\{\mathcal{Z}_{C_{m+1}, q}\right\}$ is as in $(3.2)$. This shows that the class in the Grothendieck ring of the variety $\mathcal{Z}_{(m, k)} G_{N}, q$ is in the mixed Tate subring $\mathbb{Z}[\mathbb{L}] \subset K_{0}\left(\mathcal{V}_{\mathbb{Z}}\right)$. In principle, this does not yet say that the motive of $\mathcal{Z}_{(m, k)} G_{N}, q$ is mixed Tate (in the category of mixed motives), as the class $\left[\mathcal{Z}_{(m, k)} G_{N}, q\right]$ determines only its motivic Euler characteristic $\chi_{m o t}\left(\mathcal{Z}_{(m, k) G_{N}, q}\right)$. However, if the conjecture holds, which predicts that knowledge of the counting function $N_{\mathfrak{p}}$ suffices to determine the motive, then we can conclude from the expression above for the Grothendieck class that the motive is mixed Tate. Then the main result of 13 shows that the period 6.3 has to be a combination of multiple zeta values as stated.

One can then ask a more precise question.

Question 6.2. What combinations of multiple zeta values arise in the period computations (6.3) for the polymer chain graphs ${ }^{(m, k)} G_{N}$ ? How do they behave as the parameters $m, k, N$ get large?

With respect to this last question, and the more general question of identifying criteria for when a graph satisfies the polynomial countability condition, it is worth mentioning another important difference between the quantum field theory case and the Potts model hypersurfaces.

In the case of the graph hypersurfaces of quantum field theory, where the hypersurface is defined by the vanishing of the Kirchhoff polynomial, one can use the matrix-tree theorem to obtain some more precise information on the periods. In fact, the Kirchhoff polynomial can be written as $\Psi_{G}=\operatorname{det}\left(\mathcal{M}_{G}\right)$, where $\mathcal{M}_{G}$ is a matrix defined in terms of the combinatorics (the incidence data) of the graph. One then has algebraic relations between determinants of minors of the matrix $\mathcal{M}_{G}$, which define relations between the corresponding Dodgson polynomials $\Psi_{G}^{I, J}$ of the graph. These identities were used in [14 to identify structural properties of graphs whose associated Feynman period is a multiple zeta value. This identifies a "linear reducibility criterion" for graphs, which implies the mixed-Tate property. Another setting in which the matrix-tree theorem for Kirchhoff polynomial can be useful in the quantum field theory case is the result of [5], where one uses the determinant description of the Kirchhoff polynomial to map the Feynman integral computation to the complement of a determinant hypersurface. In the case of the multivariate Tutte polynomials, however, it is well known that one does not have an analog of the matrix-tree theorem. In fact, the problem of computing the Tutte polynomials is known to be \#P-hard, see [26].

6.1. Potts models on tetrahedral chains. Our previous observations on the tetrahedron graph suggest that the same approach we just described for polygonal polymer chains may lead to possibly very different answers when applied to a different type of chain graphs, the tetrahedral chains that arise naturally in the study of silicates.

The simplest example of Potts model, the Ising model with $q=2$ spin states, has been extensively used in studying the properties of tetrahedral chains that arise in 


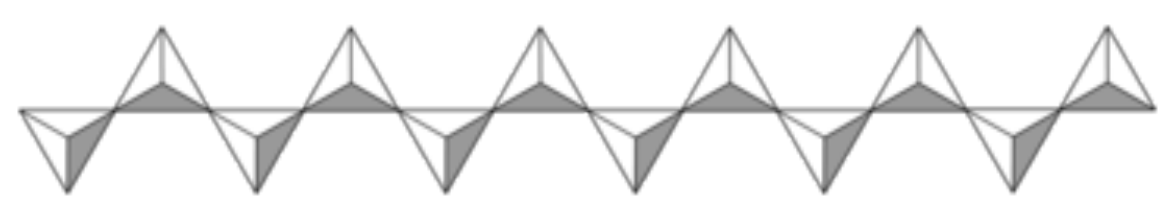

Figure 2. Tetrahedra in a single-chain configuration.

silicates and $\mathrm{Si}-\mathrm{Al}$ minerals (see $\S 6$ of $[30$ ). Example of such Ising model analysis can be found in [19], [21].

Here we consider the type of single chain tetrahedral configuration, as illustrated in Figure 2. This is realized, for instance, by inosilicates such as $\left(\mathrm{Si}_{3}^{2-}\right)_{n}$.

Recall that, for a graph $G$ that is obtained as a chain of graphs $G_{1}, \ldots, G_{N}$, where $G_{i}$ and $G_{i+1}$ have a single vertex in common, the classes in the Grothendieck ring of the corresponding Potts model hypersurface complements (with $q \neq 0$ ) is simply a product (see Lemma 3.5 of [1])

$$
\left\{\mathcal{Z}_{G, q}\right\}=\prod_{i=1}^{N}\left\{\mathcal{Z}_{G_{i}, q}\right\} .
$$

Thus, if $G_{N}$ is the graph obtained by linking together $N$ copies of the tetrahedron graph $K 4$, as in Figure 2, the corresponding class for $q \neq 0$ is given by $\left\{\mathcal{Z}_{G_{N}, q}\right\}=$ $\left\{\mathcal{Z}_{K 4, q}\right\}^{N}$, or equivalently

$$
\left[\mathcal{Z}_{G_{N}, q}\right]=\mathbb{L}^{6 N}-\left(\mathbb{L}^{6}-\left[\mathcal{Z}_{K 4, q}\right]\right)^{N},
$$

which implies that the counting function satisfies

$$
N_{\mathfrak{p}}\left(\mathcal{Z}_{G_{N}, q}\right)=\mathfrak{p}^{6 N}-\left(\mathfrak{p}^{6}-N_{\mathfrak{p}}\left(\mathcal{Z}_{K 4, q}\right)\right)^{N},
$$

at primes $\mathfrak{p}$ that do not divide $q$. In the case of the Ising model, where $q=2$, the counting function $N_{\mathfrak{p}}\left(\mathcal{Z}_{K 4, q=2}\right)$ is the one computed in (5.11).

Thus, one sees that, unlike the case of the polymer chains analyzed in [1, that involved the polynomial counting functions of polygons, here the counting function involves non-polynomial expressions, as shown in Proposition 5.8. Although this does not prove that the motive is non-mixed Tate, as there could be cancellations coming from the other terms in (5.11), it appears to be a good candidate to test. So one can ask the following question.

Question 6.3. Let $G_{N}$ be the graph obtained by linking together $N$ tetrahedra as in Figure 2. Are there choices of polynomials $F(t)$ with rational coefficients, for which the period 6.3 , computed for this graph is not a $\mathbb{Q}\left[\frac{1}{2 \pi i}\right]$-linear combinations of multiple zeta values? What kind of period is it, if that is the case?

Notice that the analogous question in the quantum field theory setting is not yet solved, even though there are explicit non-mixed Tate examples of graph hypersurfaces as in [16. In fact, there is not yet an explicit example of graph hypersurface for which the Feynman amplitude itself would be a non-mixed Tate period, although there are good reasons to expect that, as explained in [16]. 
Acknowledgment. This paper is based on the results of the second author's summer research project, supported by the Summer Undergraduate Research Fellowship program at Caltech. The first author is partly supported by NSF grants DMS-0901221 and DMS-1007207. The authors thank Paolo Aluffi and Bill Dubuque for useful conversations.

\section{REFERENCES}

[1] P. Aluffi and M. Marcolli, A Motivic approach to phase transitions in Potts models, arXiv:1102.3462 1 [math-ph]

[2] P. Aluffi and M. Marcolli, Graph hypersurfaces and a dichotomy in the Grothendieck ring, Lett. Math. Phys. Vol.95 (2011) 223-232.

[3] P. Aluffi and M. Marcolli, Feynman motives of banana graphs. Commun. Number Theory Phys. 3 (2009), no. 1, 1-57.

[4] P. Aluffi and M. Marcolli, Feynman motives and deletion-contraction relations, in "Topology of algebraic varieties and singularities", 21-64, Contemp. Math., 538, Amer. Math. Soc., Providence, RI, 2011.

[5] P. Aluffi and M. Marcolli, Parametric Feynman integrals and determinant hypersurfaces, Advances in Theoretical and Mathematical Physics, Vol.14 (2010) 911-963.

[6] Y. André, An introduction to motivic zeta functions of motives, arXiv:0812.3920

[7] P. Belkale, P. Brosnan, Matroids, motives, and a conjecture of Kontsevich, Duke Math. Journal, Vol.116 (2003) 147-188.

[8] F. Bittner, The universal Euler characteristic for varieties of characteristic zero, Compos. Math. 140 (2004), no. 4, 1011-1032.

[9] J. Bjorken, S. Drell, Relativistic Quantum Fields, McGraw-Hill, 1965.

[10] S. Bloch, H. Esnault, D. Kreimer, On motives associated to graph polynomials, Comm. Math. Phys. 267 (2006), no. 1, 181-225.

[11] S. Bloch, D. Kreimer, Mixed Hodge structures and renormalization in physics. Commun. Number Theory Phys. 2 (2008), no. 4, 637-718.

[12] D.J. Broadhurst, D. Kreimer, Association of multiple zeta values with positive knots via Feynman diagrams up to 9 loops, Phys. Lett. B 393 (1997), no. 3-4, 403-412.

[13] F. Brown, Mixed Tate motives over $\mathbb{Z}$, arXiv:1102.1312 [math.AG]

[14] F. Brown, On the periods of some Feynman integrals, arXiv:0910.0114 [math.AG]

[15] F. Brown, The massless higher-loop two-point function, Commun. Math. Phys., Vol.287 (2009) 925-958.

[16] F. Brown, O. Schnetz, A K3 in $\phi^{4}$, arXiv:1006.4064 v3 [math.AG]

[17] D. Doryn, On one example and one counterexample in counting rational points on graph hypersurfaces, arXiv:1006.3533 1 [math.AG]

[18] D. Doryn, Cohomology of graph hypersurfaces associated to certain Feynman graphs, Commun. Number Theory Phys. 4 (2010), no. 2, 365-415.

[19] M.T. Dove, Theory of displacive phase transitions in minerals, American Mineralogist, Vol.82 (1997) 213-244.

[20] C.M. Fortuin, P.W. Kasteleyn, On the random cluster model. Physica (Amsterdam) 57 (1972), $536-564$

[21] F. Gény, L. Monnerie, Study of a Model of the Polyethylene Chain. Comparison of the Rotational Isomerism Theory with Results Obtained by a Simulation of the Brownian Motion, Macromolecules, Vol.10 (1977) N.5, 1003-1006.

[22] H. Gillet, C.Soulé, Descent, motives and K-theory. J. Reine Angew. Math. 478 (1996), 127176.

[23] D. Grigoriev and M. Karpinski, An approximation algorithm for the number of zeros of arbitrary polynomials over GF $[q]$, 32nd Annual Symposium on Foundations of Computer Science (San Juan, PR, 1991), 662-669, IEEE Comput. Soc. Press, Los Alamitos, CA, 1991.

[24] C. Itzykson, J.B. Zuber, Quantum Field Theory, Dover Publications, 2006.

[25] B. Jackson, A. Sokal, Zero-free regions for multivariate Tutte polynomials (alias Potts-model partition functions) of graphs and matroids. J. Combin. Theory Ser. B 99 (2009), no. 6, 869-903. 
[26] F. Jaeger, D. Vertigan, D. Welsh, On the computational complexity of the Jones and Tutte polynomials. Math. Proc. Cambridge Philos. Soc. 108 (1990), no. 1, 35-53.

[27] R. Karp, M. Luby, N. Madras, Monte Carlo approximation algorithms for enumeration problems, J. Algorithms 10 (1989), no. 3, 429-448.

[28] M. Kontsevich, D. Zagier, Periods, in "Mathematics unlimited2001 and beyond", 771-808, Springer, Berlin, 2001.

[29] M. Marcolli, Feynman motives, World Scientific, 2010.

[30] A. Putnis, Introduction to mineral sciences, Cambridge University Press, 1992.

[31] M. Reine, Counting rational points over quiver moduli, Int. Math. Res. Lett. (2006) art.70456, $1-19$.

[32] O. Schnetz, Quantum field theory over $\mathbb{F}_{q}$, Electron. J. Comb., Vol.18 (2011) N1, P102 [23 pages]

[33] O. Schnetz, Quantum periods: A census of $\phi^{4}$-transcendentals, Communications in Number Theory and Physics, 4 no. 1 (2010), 1-48.

[34] A.D. Sokal, The multivariate Tutte polynomial (alias Potts model) for graphs and matroids, Surveys in combinatorics 2005, 173-226, London Math. Soc. Lecture Note Ser., 327, Cambridge Univ. Press, Cambridge, 2005.

[35] R.P. Stanley, Spanning trees and a conjecture of Kontsevich, Ann. Comb. 2 (1998), no. 4, 351-363.

[36] J.R. Stembridge, Counting points on varieties over finite fields related to a conjecture of Kontsevich, Ann. Comb. 2 (1998), no. 4, 365-385.

Mathematics Department, Mail Code 253-37, Caltech, 1200 E. California Blvd. Pasadena, CA 91125, USA

E-mail address: matilde@caltech.edu

E-mail address: jessicas@caltech.edu 